\title{
Designing a National Strategy to Enable Human-Relevant Research in India: A Multistakeholder Roundtable Meeting Report
}

doi:10.14573/altex.2111151

\section{Introduction}

The last few decades have seen a global increase in the development of non-animal and human-relevant technologies, i.e., new approach methodologies (NAMs), which include in vitro and computational tests, and their respective regulatory implementation. However, the field of NAMs is still nascent in India, where these methods have only started to be used within the last five years (Parvatam et al., 2020) but have already achieved some changes in Indian regulations and guidelines (Pant, 2020). An 2016 amendment to India's Drugs and Cosmetics Rules recognized non-animal tests for dermal and ocular toxicity (Drugs and Cosmetics (Seventh Amendment) Rules, 2016). The Indian Pharmacopeia Commission, which sets standards for all drugs manufactured, sold and consumed in India, abolished the abnormal toxicity test on animals and introduced alternatives to the rabbit pyrogen test in 2020. Provisions for minimizing animal testing and acceptance of non-animal approaches were incorporated in a recently released guideline for the evaluation of nano-based agri-input (feed, fertilizers and plant protection products) and food products in India (DBT, 2020). In addition, the updated guidance document on the toxicology required for registration of chemical pesticides now recognizes the use of in vitro tests and non-testing approaches such as waivers, read-across (data bridging) and valid in silico models (MOAFW, 2017).

Apart from regulatory changes in the areas of toxicology and drug development, there have also been several initiatives towards promoting the use of NAMs in biomedical research in India. In 2019, a perspectives paper by the Indian Council of Medical Research (ICMR) highlighted the need for non-animal-based research in India (Swaminathan et al., 2019). In addition, ICMR also drafted a white paper that proposed an Indian roadmap for alternatives to animals in research. There has been a significant increase in the number of research labs and start-ups that use or develop in vitro microphysiological systems (MPS) models for basic biological research in the last few years (Parvatam et al., 2020).

These research and government initiatives indicate that this is an opportune moment to accelerate non-animal and human-relevant research in India. However, there are certain roadblocks to achieving these goals. These were discussed at a recent meeting with academia and industry stakeholders involved in NAM development, including MPS and computational models (Parvatam et al., 2021). These include lack of infrastructure, lack of a skilled pool of researchers trained in NAM methodologies, supply chain blocks, lack of awareness amongst academic and government bodies, bottlenecks in cell sourcing, and lack of communication between technology developers, regulatory bodies, and end-users.

To address these issues, the Centre for Predictive Human Model Systems (CPHMS) at Atal Incubation Centre-Centre for Cellular and Molecular Biology (AIC-CCMB) organized a multi-stakeholder roundtable meeting between MPS technology developers (in academia and industry), regulators, policy-makers, and personnel from pharma companies (Tab. S1 ${ }^{1}$ ). CPHMS was established in collaboration between AIC-CCMB and the Humane Society International (India) to advance and enable human-relevant methodologies in India. This roundtable meeting was a first-of-its-kind, cross-sectoral meeting in India to discuss the development of MPS technology. The discussion was focused on two themes: indigenous development of MPS models and developing a sustained source of funding for these methods. Several recommendations were proposed by the members of the government, regulatory, and funding bodies during the meeting as discussed below.

\section{Recommendations to develop non-animal, human-relevant methodologies in India}

\subsection{Building a database to map MPS research in India}

As MPS research is still new in India, it was suggested to build an open-access, interactive database to map the researchers, their skills, and the developed technologies. Such a database would assist not just in mapping the expertise and work being done in different areas of research but would also document the gaps in training and infrastructure. It would also help in forging collaborations, reducing redundancy, and identifying specific technologies that are ahead in terms of their technology readiness levels. Armed with this information, funding could be directed towards specific gap areas in training, research, and infrastructure that are relevant to India.

1 doi:10.14573/altex.2111151s 


\subsection{Building Centres of Excellence for human-relevant research}

In the past five years, more than 30 research labs and start-ups initiated work in the area of MPS research in India (Parvatam et al., 2020). This research spans diverse organ systems, including eye, liver, brain, skin, wound infections, placenta, lung, and various cancers to understand human development and drug discovery. However, much of the research currently occurs in siloes. Building dedicated Centres of Excellence would have a multi-fold impact by bringing researchers with varied expertise and skills together to build MPS models for drug discovery. This could also accelerate the pace of research and reduce redundancy, as multiple groups are working on similar themes.

\subsection{Thematic categories in government and private grants}

Currently, researchers working on MPS projects apply to the broad categories of stem cell or disease biology for government grants. Creating thematic categories in various government and private grants could increase awareness towards this area and provide positive incentives for researchers.

\subsection{Industry-sponsored PhD or post-doctoral fellowships in this area}

To address the specific needs of industry, who are the main end-users of MPS technology in drug discovery, industry-sponsored fellowships could be provided. These fellowships could be directed towards specific needs and requirements of industry or methods identified to be at advanced stages of technology-readiness levels. This would increase the cross-talk and bridge the gap between the technology developers in academia and the end-users in pharma companies.

\subsection{Training programs and incorporation in educational curricula}

There is only a small pool of skilled researchers in India who are trained in human-relevant technologies, such as MPS models, computational tools, etc. This is due to a lack of training programs specifically geared towards this area. To address this, dedicated training programs need to be developed. To combat the lack of awareness amongst students and researchers, certificate programs or curricula could be designed for various target audiences, including students, researchers, regulators, or personnel in contract research organizations (CROs) and micro, small \& medium enterprises (MSMEs). It was also suggested that regulatory bodies be involved in these training programs, as researchers are often unaware of the process of obtaining regulatory approval for new technologies.

\subsection{Increasing public awareness and engagement}

Public opinion and civil societies can play an important role in public policy and legislative changes in a country. In India, public awareness regarding non-animal and emerging technol-

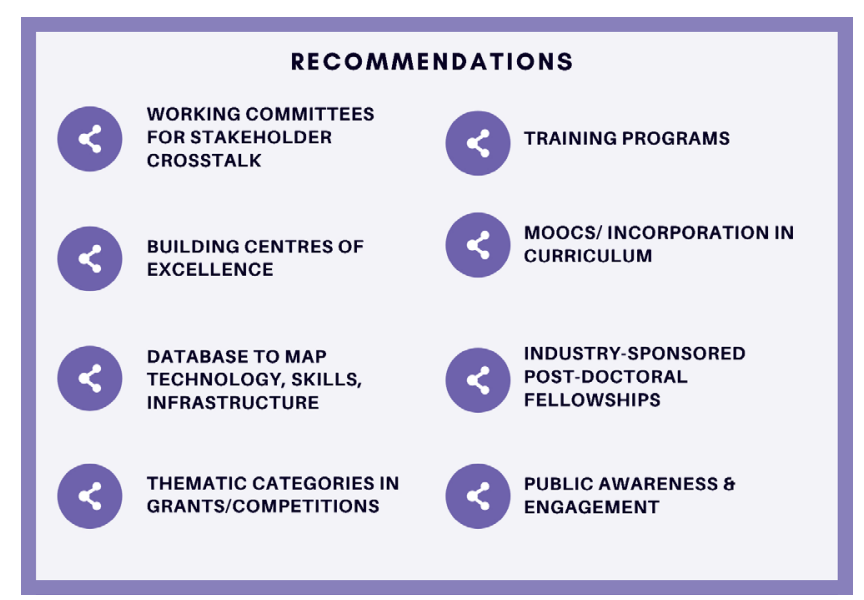

Fig. 1: Recommendations proposed in the multi-stakeholder roundtable meeting to enable the development of humanrelevant technologies in India

ogies is very low. Thus, programs that provide information in an attractive and accessible manner can be designed to engage with the public such that they can be involved in implementing change at a larger scale.

\subsection{Encouraging bilateral and multi-lateral collaborations}

Many countries are presently at advanced stages of development of these technologies. It was suggested that as India has bilateral relationships with almost 20 countries, countries with technological expertise in this area could be identified to develop specific programs for knowledge exchange.

\subsection{Increasing stakeholder crosstalk}

One of the critical lacunae is an absence of communication between technology developers in academia and industry along with the stakeholders in government, regulatory bodies, and pharma companies. This leads to gaps in understanding the requirements of end-users during the technology development and of regulators for obtaining regulatory approval. On the other hand, end-users and regulatory bodies also need to be informed regarding the technological advances in the drug discovery area. One-day multi-stakeholder workshops or meets conducted twice a year could assist in addressing this limitation.

\section{The way forward}

To take forward the recommendations of the meeting participants into actional outcomes, we propose two strategies. In 2013, India introduced the Companies Act, which made it mandatory for firms with a net worth exceeding ₹500 crore or a turnover exceeding ₹ 1,000 crore or net profit above ₹ 5 crore to spend

\footnotetext{
2 https://www.thehindu.com/business/firms-can-use-csr-funds-for-rd/article29471805.ece
} 
$2 \%$ of their average net profit on corporate social responsibility (CSR) activities. In 2019, the Government of India amended this Act such that corporations can now use their CSR funds to support research efforts in science, technology, medicine, and engineering at major institutions and bodies ${ }^{2}$. This paved the way to increasing private investment into research. For many of the recommendations proposed, including establishing Centres of Excellence, tapping into the CSR funds could provide a potent method to increase private investment and involve various industry stakeholders in developing MPS models.

As several members also suggested the formation of steering committees to carry forward these recommendations, we propose the formation of three working groups (WGs) in the areas of education and training, basic and applied research, and translational research of MPS technologies. The education and training WG would focus on building a pool of researchers and students that are skilled in NAMs. The basic research WG would focus on how to address the challenges related to research, funding, and infrastructure for developing MPS technology in India. The translational WG would address how to take the technology to end-users, including crossing regulatory hurdles and scaling up. These WGs would ideally be multi-stakeholder and could meet on a bi-annual basis to share updates and design strategies.

We envision that these initiatives will commence in the coming few months and assist in achieving the goals proposed at this roundtable meeting.

\section{References}

DBT - Department of Biotechnology (2020). Guidelines for Evaluation of Nano-Based Agri-Input and Food Products in
India. https://static.pib.gov.in/WriteReadData/userfiles/Nano Agri_15.6.2020.pdf

Drugs and Cosmetics ( $7^{\text {th }}$ Amendment) Rules (2016). The Gazette of India. Section 148-C, Prohibition of Testing of Cosmetics on Animals, in the Drugs and Cosmetics Rules 1945. http://egazette.nic.in/WriteReadData/2014/159614.pdf

MOAFW - Ministry of Agriculture \& Farmers Welfare (2017). Guidance Document on Toxicology for Registration of Chemical Pesticides in India. http://ppqs.gov.in/sites/default/files/ toxguidancedocsept2017.pdf

Pant, A. B. (2020). The Implementation of the three Rs in regulatory toxicity and biosafety assessment: The Indian perspective. Altern Lab Anim 48, 234-251. doi:10.1177/ 0261192920986811

Parvatam, S., Bharadwaj, S., Radha, V. et al. (2020). The need to develop a framework for human-relevant research in India: Towards better disease models and drug discovery. J Biosci 45, 144. doi:10.1007/s12038-020-00112-8

Parvatam, S., Bharadwaj, S. and Poojary, B. (2021). Enabling a shift towards a human-relevant and predictive paradigm for biomedical research and drug discovery in India. ALTEX 38, 507-509. doi:10.14573/altex.2103221

Swaminathan, S., Kumar, V. and Kaul, R. (2019). Need for alternatives to animals in experimentation: An Indian perspective. Indian J Med Res 149, 584-592. doi:10.4103/ijmr. IJMR_2047_17

\section{Surat Parvatam}

Centre for Predictive Human Model Systems, Atal Incubation Centre-

Centre for Cellular and Molecular Biology, Hyderabad, India 